\title{
Emergency Panic Button using Microcontrollers
}

\author{
Nivedita Majumdar \\ School of Computing Science \\ and Engineering \\ VIT University, Vellore \\ Tamil Nadu, India
}

\author{
Pragati Bhargava \\ School of Computing Science \\ and Engineering \\ VIT University, Vellore \\ Tamil Nadu, India
}

\author{
Rubeena K Shirin \\ School of Computing Science \\ and Engineering \\ VIT University, Vellore \\ Tamil Nadu, India
}

\begin{abstract}
Safety is a primary concern today for people everywhere. No one can foresee danger, and reaction to any danger is required in a split second. Recently, mobile apps have been developed, which can be used to connect to your emergency contact. But not everyone has a smartphone. Moreover, it is not possible to use an app in a split second. Microcontrollers are used in devices everywhere. An ideal panic button should be one which allows the user to react and finish the work at the fastest speed possible, while successfully accomplishing the task. This paper describes a panic button that will ensure safety in minimal time. A simple switch will trigger the device that consists of an Arduino board and GSM Module. The Arduino board sends a signal to the GSM Module with a SIM Card, which will send an emergency message to the contact pre-defined in the system, along with the exact GPS co-ordinates of the position of the user with the panic button. This information can be used by the emergency contact to pinpoint the user's location exactly and track the user in the shortest time possible.
\end{abstract}

\section{General Terms}

Your general terms must be any term which can be used for general classification of the submitted material such as Pattern Recognition, Security, Algorithms et. al.

\section{Keywords}

Keywords are your own designated keywords which can be used for easy location of the manuscript using any search engines.

\section{INTRODUCTION}

In an ever-changing world, there are a lot of difficulties faced by people all over the world. The major concern faced by majority of the population is safety. Every situation cannot be foreseen, and every threat cannot be predicted. Senior Citizens living alone could face an emergency medical situation. Someone could break in to your home at night, or while walking down a lonely street, there is a possibility of being attacked. Women's Safety is a major source of worry in every country, while the safety of children is of equal importance. Most accidents occur due to the fact that help could not reach in time, because contact could not be established with anyone outside in a short time [1].

Recent App developers have developed mobile applications for smartphones, which instantly send messages to emergency contact, the software counterpart of a microcontroller based panic button [2]. But there are disadvantages in using a smartphone app. Firstly, there are battery problems, and if there isn't enough power in the phone, the app will not work. Second, it takes time to open an app and use it, which increases reaction time, and that could prove to be dangerous. A microcontroller based device aims to reduce reaction time so that the user can react to a threat in a jiffy.

Our Project has aimed at making a simple low cost Panic Button device which can be accessed by women across the world.

\section{SYSTEM COMPONENTS}

The panic button has various hardware and software requirements. The various Hardware components required are Arduino Uno Board, GSM Module and GPS Tracker, push button, Power Source Battery) and Connecting wires and cables. The Software requirement is Arduino software. Figure 1 shows the real time connections of the systems, when the device was assembled by us.

This section will explain the role of each component in the system.

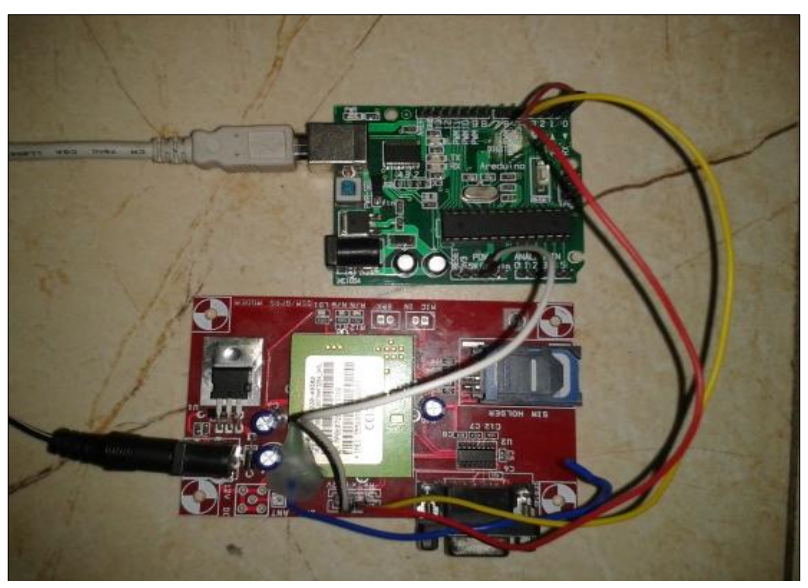

Figure 1- Real Time Connections of the System

\subsection{Arduino Uno Board}

The Arduino Uno is a microcontroller board based on the ATmega32 [3]. It has 14 digital input/output pins, 6 Analog inputs, a $16 \mathrm{MHz}$ ceramic resonator, a USB connection, a Power jack, an ICSP header, and a reset button. It contains everything needed to support the microcontroller; simply connect it to a computer with a USB cable or power it with an AC-to-DC adapter or battery to get started. 


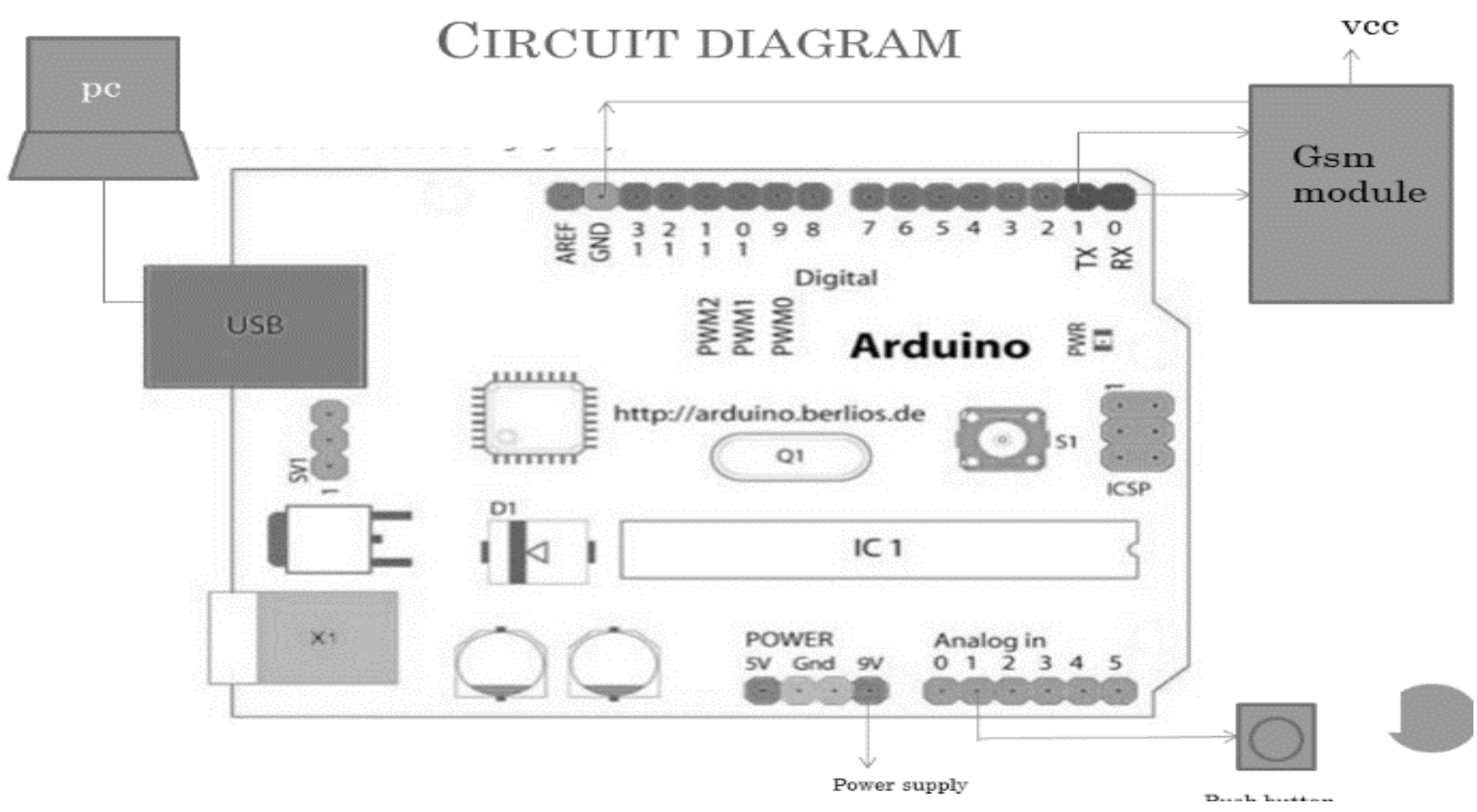

Figure 2- Circuit Diagram of the system

\subsection{GSM Module}

The connection between the Arduino board and another GSMGPRS system is established with the help of a GSM/GPRS Module [4-5]. GSM (Global System for Mobile Communication) is an architecture system mainly used for mobile communication. Global Packet Radio Service (GPRS) is an extension of GSM that enables higher data transmission rate. GSM/GPRS module consists of a GSM/GPRS modem assembled together with power supply circuit and communication interfaces. It has a SIM card holder, where the user's SIM is inserted. This card will vary according to the user's Mobile Service Provider and the message will be sent using that SIM [6].

\subsection{Push Button}

The Push Button will act as a trigger for the system. If the button is pushed, the system is activated and message is sent. For easy activation, the push button is easy to press and has a faster reaction time than normal buttons.

\subsection{Arduino Software}

Arduino Software in the computer is used to compile the required software programs and load them into the microcontroller of the Arduino Uno Board for execution. The USB connector on one end is connected to the PC with the Arduino software and the other end is connected to the USB port on the Arduino Board. Once the code for triggering the GSM Module (with a specific phone number) is compiled and ready to be executed, it is loaded onto the Arduino board. Arduino software is easy to learn and execute [7].

\section{INTERACTION OF THE DEVICE}

With all the modules ready, the next step is to connect all the modules so that there is proper interaction between the modules for smooth functioning of the system.

Figure 2 gives an accurate Circuit Diagram of the system. The diagram also shows how the components are connected.

\subsection{Software Programming}

Arduino software has to be loaded on to the system you are working from. The relevant codes for sending a message with GPS coordinates have to be compiled on the software. Once the codes are compiled and ready, connect the Arduino board to the computer system using USB cable, and copy the code into the microcontroller memory.

\subsection{Starting the GSM Module}

Insert a SIM Card in the holder provided in the GSM Shield. Connect the GSM Module to a $12 \mathrm{~V}$ battery (external power source) [8] and switch it on. A blinking red light will indicate that the SIM card has valid signal. For checking if your GSM shield is working, call the phone number associated with the SIM card. An orange continuous blinking light indicates that the SIM card is ready to accept the call and connected.

\subsection{Connecting GSM Module to Arduino Board}

Using Connecting Wires, connect the TX pin, RX Pin and GND of the GSM Module to the same exact pins on the Arduino Board. Make sure the GPS Module and the Arduino Board are switched on. The Connections are established. Figure 3 shows the real time connections in the device.

\subsection{Connecting Switch to Arduino Board}

A switch or push button is connected to the Arduino Board. In the off state of the switch, the device will be in an idle state. Once the switch is flipped, the system becomes active. The Arduino Board sends a signal to the GSM Module, which will get activated and send a message using the SIM Card attached 
in the GSM Module [9]. The message will be sent to a predefined emergency contact number specified by the user. Figure 3 is a flowchart of the Real time working of the device.

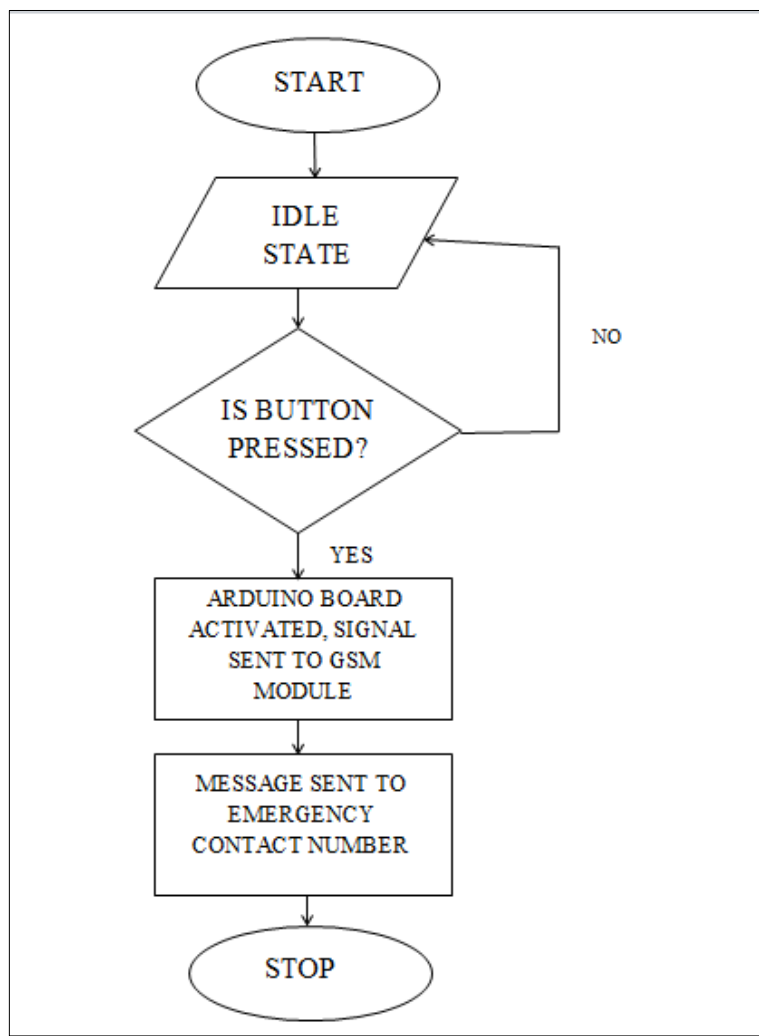

Figure 3- Flowchart of Real Time Working of the Device

\section{CONCLUSION AND FUTURE WORK}

The proposed device can be marketed as a low cost safety device, and is very purposeful and portable. The construction cost is low, and programs are readily available. Components are easily available in the market. GPS Module can be added to get the exact co-ordinates of the location of the user [10]. There is one difficulty, and that is the size. Size optimization of the device can be done further, because even though the device is portable, it needs to be reduced in size so that it can be carried the user with ease. Safety is a prime concern for all of us today, and this device ensures that a sense of security prevails in the user carrying it.

\section{ACKNOWLEDGMENTS}

This paper is a part of our program for the subject Embedded Systems, a Project Based Learning course, which is a part of the curriculum for B.Tech Computer Science and Engineering taught at VIT University, Vellore, Tamil Nadu, India.

We would foremost like to thank our Project Guide, Prof. Narayanamoorthi $M$ of School of Computing Science and Engineering at VIT University for his unending support and guidance throughout the Project.

\section{REFERENCES}

[1] Paul Gantt, Ron Gantt, "Disaster Psychology", Professional Safety, August 2012

[2] Priyanka Shinde, Pranita Taware, Snehal Thorat, Tejashree Waghmare, "Emergency Panic Button (Android App)" , International Journal of Scientific and Engineering Research (ISSN 2229-5518), Volume 3Issue 3, March 2012 .

[3] Arduino Uno Product Overview http://arduino.cc/en/Main/arduinoBoardUno

[4] Nikhil Agnihotri, Article on the working of GSM/GPRS Module, Engineers Garage

[5] Ma Yuchun, Huang Yinghong, Zhang Kun, Li Zhuang, "General Application Research in GSM Module", 2011 International Conference on Internet Computing \& Information Services

[6] Mohsen Sharakbi, GSM Module Overview http://www.slideshare.net/mohsensarakbi/gsm-module

[7] Alan G. Smith, Introduction to Arduino, http://www.princeton.edu, September 2011.

[8] Mallikarjun Sarsamba, Prashant Sangulagi, Dr. Raju Yanamshetty, "The Load Monitoring and Protection on Electricity Power lines using GSM Network", International Journal of Advanced Research in Computer Science and Software Engineering, Volume 3, Issue 9, September 2013.

[9] Boris Landoni, "How to Send and receive SMS with GSM/GPRS \& GSM Shield", open-electronics.org, May 2013

[10] S.P. Drake, "Converting GPS Coordinates to Navigation Coordinates", Surveillance Systems Division, DSTO Electronics and Surveillance Research Laboratory, DSTO-TN-0432. 